Article

\title{
On Moderate-Rayleigh-Number Convection in an Inclined Porous Layer
}

\author{
Baole Wen 1,2,*iD, Gregory P. Chini ${ }^{3,4, *}$ \\ 1 Institute of Computational Engineering and Sciences, The University of Texas at Austin, \\ Austin, TX 78712, USA \\ 2 Department of Geological Sciences, University of Texas at Austin, Austin, TX 78712, USA \\ 3 Program in Integrated Applied Mathematics, University of New Hampshire, Durham, NH 03824, USA \\ 4 Department of Mechanical Engineering, University of New Hampshire, Durham, NH 03824, USA \\ * Correspondence: wenbaole@gmail.com (B.W.); greg.chini@unh.edu (G.P.C.)
}

Received: 29 April 2019; Accepted: 28 May 2019; Published: 31 May 2019

\begin{abstract}
We investigate the flow structure and dynamics of moderate-Rayleigh-number $(R a)$ thermal convection in a two-dimensional inclined porous layer. High-resolution numerical simulations confirm the emergence of $O(1)$ aspect-ratio large-scale convective rolls, with one 'natural' roll rotating in the counterclockwise direction and one 'antinatural' roll rotating in the clockwise direction. As the inclination angle $\phi$ is increased, the background mean shear flow intensifies the natural-roll motion, while suppressing the antinatural-roll motion. Our numerical simulations also reveal-for the first time in single-species porous medium convection-the existence of spatially-localized convective states at large $\phi$, which we suggest are enabled by subcritical instability of the base state at sufficiently large inclination angles. To better understand the physics of inclined porous medium convection at different $\phi$, we numerically compute steady convective solutions using Newton iteration and then perform secondary stability analysis of these nonlinear states using Floquet theory. Our analysis indicates that the inclination of the porous layer stabilizes the boundary layers of the natural roll, but intensifies the boundary-layer instability of the antinatural roll. These results facilitate physical understanding of the large-scale cellular flows observed in the numerical simulations at different values of $\phi$.
\end{abstract}

Keywords: convection; porous media; secondary stability; floquet theory; localized states

\section{Introduction}

Buoyancy-driven convection in fluid-saturated porous media exhibits rich instability characteristics and nonlinear dynamics as the Rayleigh number $R a$, a dimensionless parameter characterizing the ratio of driving to damping forces, increases [1-7]. This system has been extensively studied owing to applications in geothermal energy extraction, geological carbon sequestration, and the the design of compact heat exchangers [8-11]. In a homogenous and isotropic horizontal porous layer uniformly heated from below, the basic conduction state becomes linearly unstable above a critical Rayleigh number $R a_{c}=4 \pi^{2}$ [1,2], giving rise to steady $O(1)$ aspect-ratio large-scale convective rolls through a stationary bifurcation. As $R a$ is increased further, a secondary instability occurs within the upper and lower thermal boundary layers via a supercritical Hopf bifurcation, generating small-scale plumes that are periodically or quasi-periodically advected around the cells for $400 \lesssim R a \lesssim 1300$ [3,12-16]. For $R a>1300$, the large-scale cellular flow is broken down and the system transitions to the 'turbulent', narrowly spaced columnar-flow, high- $R a$ regime [4-6,17].

In deep geological formations the layer may not be strictly horizontal; for example, in carbon sequestration the saline aquifers are generally inclined at an angle to the horizontal [18-21]. 
The inclination of the layer introduces an additional control parameter, i.e., the tilt angle, which significantly affects the instability and bifurcation of the base flow. In a sloping three-dimensional (3D) porous layer with an inclination angle $\phi$ above the horizontal, four types of flows exist near the onset of convection: the basic single-cell shear flow with an upward current near the lower heated wall and a downward current near the upper cooled wall (Figure 1); polyhedral cells with wall-normal axes; longitudinal helicoidal cells resulting from the longitudinal rolls (with wall-parallel axes) superposed on the basic flow; and two-dimensional (2D) transverse rolls [22-35]. Note that in an infinitely extended layer, the unicellular base state becomes independent of the wall-parallel $(x)$ coordinate and reduces to a laminar unidirectional shear flow, as schematically depicted in Figure 1 . The early experiments by Bories and collaborators [22-24] indicated that the basic unicellular flow is stable for $R a \cos \phi \leq 4 \pi^{2}$; when $R a \cos \phi$ is slightly greater than $4 \pi^{2}$, however, convection appears in the form of polyhedral cells for small inclination angles $\left(\phi \lesssim 15^{\circ}\right)$ and longitudinal helicoidal rolls for larger $\phi$. Besides these three flow configurations, 2D transverse rolls are also observed at small $R a$ and $\phi$, e.g., in the experiments by Caltagirone et al. [25], Kaneko [26], and Kaneko et al. [27], and in the numerical simulations by Caltagirone and Bories [28].

In order to investigate the conditions for transitions between these different flow regimes, a series of subsequent studies were carried out. Using linear stability analysis, Caltagirone and Bories [28] demonstrated that in an infinitely extended porous layer, the basic-state shear flow is stable for $R a \cos \phi \leq 4 \pi^{2}$. These authors also obtained a transition criterion from the polyhedric cells or transverse rolls to the helicoidal cells, with their analysis yielding a transition angle $\phi_{t} \simeq 31.8^{\circ}$ between these flow patterns. More recently, a full numerical investigation of the marginal stability of the background flow was performed by Rees and Bassom [29] in a 2D inclined porous layer. Since all fields are presumed to be independent of the transverse $(y)$ direction, polyhedral cells and helicoidal rolls cannot be realized in the 2D layer. Consequently, the basic unicellular flow can be linearly stable at smaller $\phi$. Moreover, as shown in Reference [29], 2D linear instability at large Ra can only arise when $\phi \leq 31.3^{\circ}$. Additional linear stability analyses have been performed with the aim of understanding the effects of material anisotropy and variations in boundary conditions [30-35]. Crucially, recent analysis by Wen and Chini [36] indicates that the basic state is not energy stable for $\phi \leq 90^{\circ}$ and $R a>91.6$, so this base state may become unstable to sufficiently large-amplitude disturbances for $\phi>31.3^{\circ}$.

Instead of focusing on the onset of convection, in this work we numerically investigate how layer inclination affects the flow structure and dynamics of finite-amplitude convection at moderate values of the Rayleigh number $(R a<1000)$. Although some numerical simulations of porous medium convection have been performed in inclined cavities to investigate the emergent steady convective flow at small $R a$ [37-39], the side walls may significantly impact the flow structure and transport properties if the aspect ratio of the domain is not sufficiently large (e.g., in a sloping square cavity). Here, we conduct well-resolved numerical simulations in an inclined 2D Rayleigh-Darcy domain having $O(1)$ aspect ratio but enforce periodicity rather than sidewall conditions in the wall-parallel $(x)$ direction, since the former enables a better approximation of the physics of convection in an extended layer. Of course, in the moderate- $R a$ regime on which we focus, the convection is not 'turbulent' but rather spatially coherent, implying that the quantitative results of our simulations necessarily will depend on the precise $O(1)$ value of the aspect ratio employed. Nevertheless, we expect that the observed trends, qualitative results, and physical insights gleaned from our simulations will be robust to variations in the domain aspect ratio. Moreover, we perform one set of simulations in an extended domain of large aspect ratio to investigate, for the first time in single-species porous media convection, the phenomenon of spatial localization of the emergent convection patterns. To elucidate the physical mechanisms manifested in the simulations as $R a$ and $\phi$ are varied, we also compute (dynamically unstable) steady convective solutions using Newton iteration and then perform secondary stability analysis of these nonlinear states numerically using Floquet theory. Collectively, our results shed light on the development of moderate- $R a$ large-scale cellular flows at different inclination angles. 


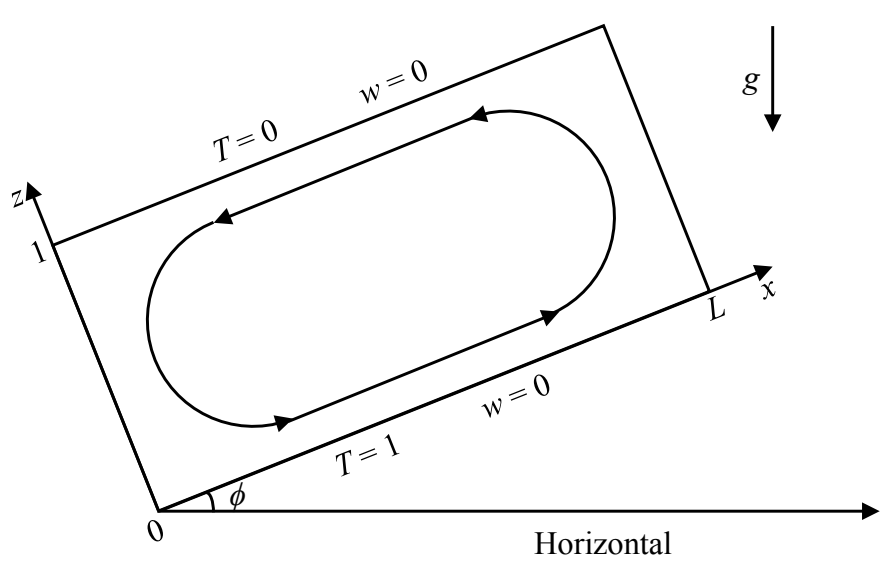

(a)

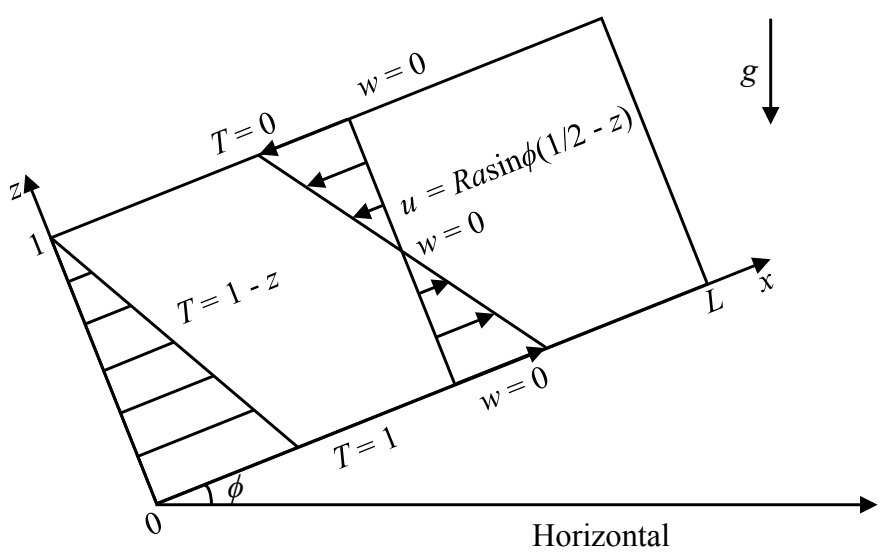

(b)

Figure 1. Dimensionless geometry and background/basic state for $2 \mathrm{D}$ convection in inclined Rayleigh-Darcy domains. (a) Closed domain; (b) L-periodic domain. $x$ and $z$ are the wall-parallel and wall-normal coordinates, respectively, and $g$ is the (dimensional) acceleration of gravity. For $\phi>0^{\circ}$, the basic-state temperature field (realized in the absence of convection) varies only in $z$, as in the horizontal case $\left(\phi=0^{\circ}\right)$. The basic-state velocity field is nonzero, however, with the background shear flow strengthening as the inclination angle $\phi$ is increased. The flow in (a) represents the basic unicellular shear flow observed in experiments in a closed domain. In an infinitely extended layer (i.e., $L \rightarrow \infty$ ), the unicellular base state becomes $x$-independent and reduces to a laminar unidirectional shear flow, as shown in (b).

The remainder of this paper is organized as follows. In the following section, we formulate the standard mathematical model of inclined porous medium convection. In Section 3, we perform numerical simulations in the moderate- $R a$ regime for a range of inclination angles, and then investigate the structure and stability of steady nonlinear convective states. Finally, our conclusions are given in Section 4.

\section{Governing Equations}

Consider a 2D fluid-saturated porous layer inclined at an angle $\phi$ above the horizontal (Figure 1). The domain is heated from below and has aspect ratio $L$. We assume the motion of the incompressible fluid satisfies the Boussinesq approximation and Darcy's law. The system is rendered dimensionless using the layer thickness $H$, the temperature difference across the layer $\Delta T$, the diffusion time $\mathcal{T}_{d}=\alpha_{m} H^{2} / \kappa$, and the diffusion velocity $U_{d}=\kappa / H$, where $\alpha_{m}$ is the ratio between the overall volumetric heat capacity of the porous media and the volumetric heat capacity of the fluid, and $\kappa$ is 
the thermal diffusivity $[10,29,31-35]$. Then, the flow and heat transport processes of the system are governed by the following non-dimensional equations [10]:

$$
\begin{gathered}
\nabla \cdot \mathbf{u}=0 \\
\mathbf{u}+\nabla p=\operatorname{RaT}\left(\sin \phi \mathbf{e}_{x}+\cos \phi \mathbf{e}_{z}\right), \\
\partial_{t} T+\mathbf{u} \cdot \nabla T=\nabla^{2} T
\end{gathered}
$$

where $\mathbf{u}(\mathbf{x}, t)=(u, w), T(\mathbf{x}, t)$ and $p(\mathbf{x}, t)$ are the dimensionless velocity, temperature, and pressure, respectively; $\mathbf{e}_{x}$ and $\mathbf{e}_{z}$ are unit vectors in the wall-parallel ( $x$ ) and wall-normal ( $z$ ) directions; and $\nabla^{2}$ is the 2D Laplacian operator. The system of equations is solved subject to the boundary conditions

$$
T(x, 0, t)=1, \quad T(x, 1, t)=0, \quad w(x, 0, t)=0, \quad w(x, 1, t)=0 .
$$

As discussed in Section 1, all fields are required to be $L$-periodic in $x$ (Figure 1b). For the 2D system, the fluid velocity can be described by using a stream function $\psi$, so that $(u, w)=\left(\partial_{z} \psi,-\partial_{x} \psi\right)$. Then Equations (2) and (3) can be re-expressed as

$$
\begin{gathered}
\nabla^{2} \psi=R a\left(\partial_{z} \theta \sin \phi-\sin \phi-\partial_{x} \theta \cos \phi\right) \\
\partial_{t} \theta+\partial_{z} \psi \partial_{x} \theta-\partial_{x} \psi \partial_{z} \theta=-\partial_{x} \psi+\nabla^{2} \theta
\end{gathered}
$$

where $\theta(\mathbf{x}, t)=T(\mathbf{x}, t)-(1-z)$, and $\theta$ and $\psi$ satisfy $L$-periodic boundary conditions in $x$ and homogeneous Dirichlet boundary conditions in $z$.

Three dimensionless parameters control the dynamics of this system: the inclination angle $\phi$; the domain aspect ratio $L$; and the normalized temperature drop across the layer, namely, the Rayleigh-Darcy number

$$
R a=\frac{H K g \alpha \Delta T}{\kappa \nu},
$$

where $K$ is the medium permeability, $g$ is the gravitational acceleration, $\alpha$ is the thermal expansion coefficient, and $v$ is the kinematic viscosity. In an infinitely extended layer, the inclination of the domain will induce a background shear flow which strengthens as $\phi$ is increased; the corresponding basic state is defined by $T=1-z, \mathbf{u}=R a \sin \phi\left(\frac{1}{2}-z\right) \mathbf{e}_{x}$, and $p=\frac{1}{2} R a \sin \phi x+R a \cos \phi\left(z-\frac{1}{2} z^{2}\right)$ and is shown schematically in Figure $1 \mathrm{~b}$. In the next section, we demonstrate that the background shear flow dramatically impacts the flow structure as $\phi$ is increased.

\section{Dynamics at Moderate $R a$}

\subsection{Numerical Simulation Results}

In this section, high-resolution numerical simulations are performed to investigate the dynamics of convection at moderate $R a$ in an inclined porous layer. We solve Equations (5) and (6) numerically using a Fourier-Chebyshev-tau pseudospectral solver developed in References [36,40-42]. The system is discretized spatially using Fourier series in $x$ and Chebyshev series in $z$ [43], and temporally using a third-order-accurate semi-implicit Runge-Kutta scheme for the first three time steps [44] and a four-step fourth-order-accurate semi-implicit Adams-Bashforth/Backward-Differentiation scheme for all subsequent time steps [45]. The simulations are performed in domains with spatial period $L=2$ or $L=10$, where $L=2$ corresponds to the wavelength of the marginal mode for onset of convection at $R a_{c}=4 \pi^{2}$ in the horizontal case $\left(\phi=0^{\circ}\right)$. At $L=2$ and for each $R a$, the results from simulations performed at smaller $\phi$ are utilized as the initial conditions for simulations at larger $\phi$; at $L=10$ and $\phi=35^{\circ}$, however, particularly-designed and random initial conditions are used, respectively, for simulations at $R a=100$ and $R a \geq 300$. 
At small $R a$ (just above the onset of convection), the flow exhibits steady stable $O(1)$ aspect-ratio large-scale convective rolls when the layer is inclined. As shown in Figure 2, for $R a=100$ and $L=2$ there exist two steady cells corresponding to counter-rotating convective rolls: the counterclockwise circulation with positive $\psi$ and the clockwise circulation with negative $\psi$, hereafter referred to as 'natural' and 'antinatural' convective rolls, respectively. Either of these two types of steady circulation may exist in isolation in the small-aspect-ratio sloping porous cavity due to the effect of thermally-insulating lateral walls [38,39]; however, in a periodic domain, these two rolls always coexist. Moreover, for the horizontal case $\left(\phi=0^{\circ}\right)$, the steady flow exhibits centro-reflection symmetry (Figure 2a). Reflection symmetry in $x$ is broken by the layer inclination $\left(0^{\circ}<\phi<90^{\circ}\right)$, although centrosymmetry is retained (Figure $2 b, c)$. Our numerical simulation results indicate that the inclination of the layer modifies the boundary layer thickness of the velocity field for the natural and antinatural rolls: the former becomes thinner while the latter becomes thicker. Furthermore, the extremum $\psi$ value of the natural roll becomes larger as $\phi$ is increased (see the colorbar limits in Figure 2), in contrast to that of the antinatural roll, implying that compared with antinatural convective motion, the natural convective motion becomes more vigorous when the layer is inclined. This result accords with the physical intuition that, for $0^{\circ}<\phi<90^{\circ}$, the base shear flow $\mathbf{u}=R a \sin \phi\left(\frac{1}{2}-z\right) \mathbf{e}_{x}$, which intensifies for increasing $\phi$, enhances (suppresses) fluid motions with the same (opposite) sense of rotation.

As for horizontal convection, the steady rolls computed at different $\phi$ strengthen but remain stable as $R a$ is increased up to 200. As shown in Figure 3, however, at $R a=300$ the antinatural roll becomes unstable first for $\phi \gtrsim 10^{\circ}$ (while the natural roll remains stable) and small-scale proto-plumes are generated from the upper and lower thermal boundary layers and advected around the cell by the background roll (Figure 3c). Moreover, this boundary layer instability becomes much stronger as the inclination angle is increased so that the unsteady two-cell (one natural and one antinatural) convective roll pattern is split into a stable steady four-cell convective state at $\phi \approx 25^{\circ}$, as shown in Figure 3d.

For $R a \gtrsim 400$, the steady convective rolls become unstable even at small $\phi$, and the resulting flow exhibits a series of transitions between periodic and quasi-periodic roll motions (Figure 4), as observed in the horizontal case. A primary difference between inclined and horizontal porous medium convection is that the inclination of the layer alters the symmetry of the flow by intensifying the near-wall instability of the antinatural roll (associated with a thickening of the velocity boundary layer) while stabilizing the natural roll (associated with a thinning of the velocity boundary layer). As $\phi$ is increased, the boundary-layer instability of the antinatural roll becomes more vigorous so that the plumes generated from the thermal boundary layers split the original two-cell convection into multiple-cell convection, as shown in Figure 4. It is worth noting that as Ra is increased, the value of $\phi$ at which the flow transitions from two-cell convection to four-cell convection decreases (Table 1), e.g., for $R a=300,500$ and 998 , the approximate transition angle is decreased from $25^{\circ}$ to $15^{\circ}$ and finally to $5^{\circ}$.

The 2D numerical simulations performed by Caltagirone and Bories [28] and Moya et al. [38] did not exhibit convective flows at large $\phi$ in wide domains (e.g., $L=10$ ), in apparent agreement with the prediction that the basic state is linearly stable for $\phi>\phi_{t}$ with $\phi_{t} \approx 31.3^{\circ}$ [29]. Nevertheless, the basic state may become unstable when disturbance amplitudes are sufficiently large since, as shown by Wen and Chini [36], the base state is not energy stable for $\phi \leq 90^{\circ}$ at $R a>91.6$. Figure 5 shows snapshots of isotherms from numerical simulations at $\phi=35^{\circ}$ and $L=10$ for different Rayleigh numbers ranging from 100 to 500. Interestingly, not only do convective flows arise but, given different initial conditions, these convective flows can adopt distinct forms. For instance, at $R a=100$, the flow can exhibit stable localized convective structures with various numbers of roll pairs (Figure $5 a, b$ ) or large-scale cellular flows (Figure 5c); a flow pattern consisting of five replicas of the stable two-cell convective state obtained for $L=2$ (Figure $5 \mathrm{~d}$ ) is also realizable. We note that spatially-localized states previously have been observed in double-diffusive convection in porous media [46,47], but here our numerical simulations reveal—for the first time in single-species porous medium convection-the 
existence of these localized convective states at large $\phi$. Moreover, our simulation results also indicate that the (large-scale) localized roll pattern still appears instantaneously at higher $R a$ when the flow becomes unsteady (Figure 5e,f). Although the flow structure for $\phi>\phi_{t}$ at small and moderate $R a$ will not be discussed in further detail in this study, we comment that the observed spatially-localized convective states appear to arise through a subcritical bifurcation of the basic state enabled by the gap in parameter values for linear and nonlinear stability [36].

Table 1. Approximate angle $\phi$ at which the flow transitions from two-cell convection to four-cell convection in numerical simulations at moderate $R a$ and $L=2$.

\begin{tabular}{ccccc}
\hline$R \boldsymbol{a}$ & $\mathbf{3 0 0}$ & $\mathbf{5 0 0}$ & $\mathbf{7 9 2}$ & $\mathbf{9 9 8}$ \\
\hline$\phi$ & $25^{\circ}$ & $15^{\circ}$ & $10^{\circ}$ & $5^{\circ}$ \\
\hline
\end{tabular}
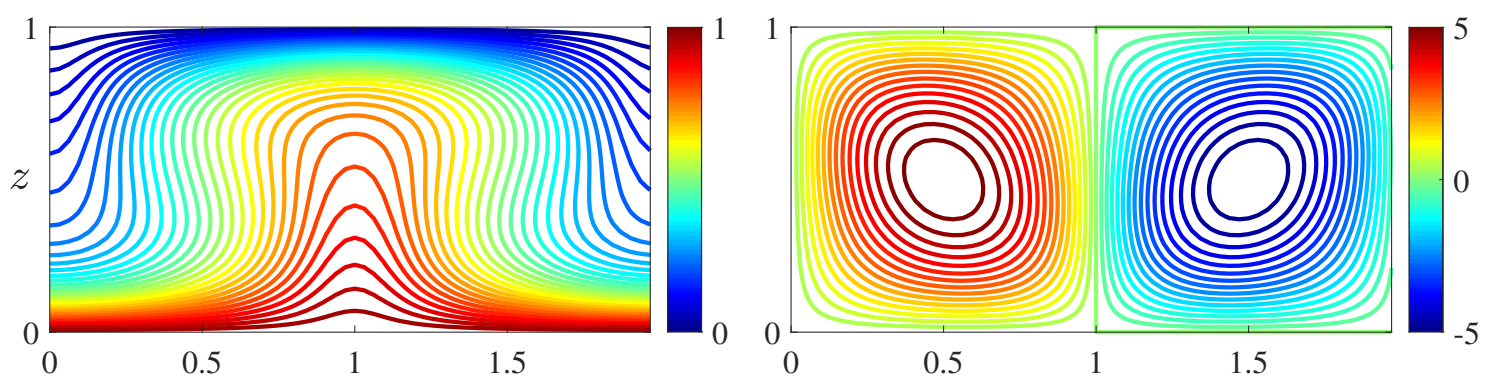

(a)
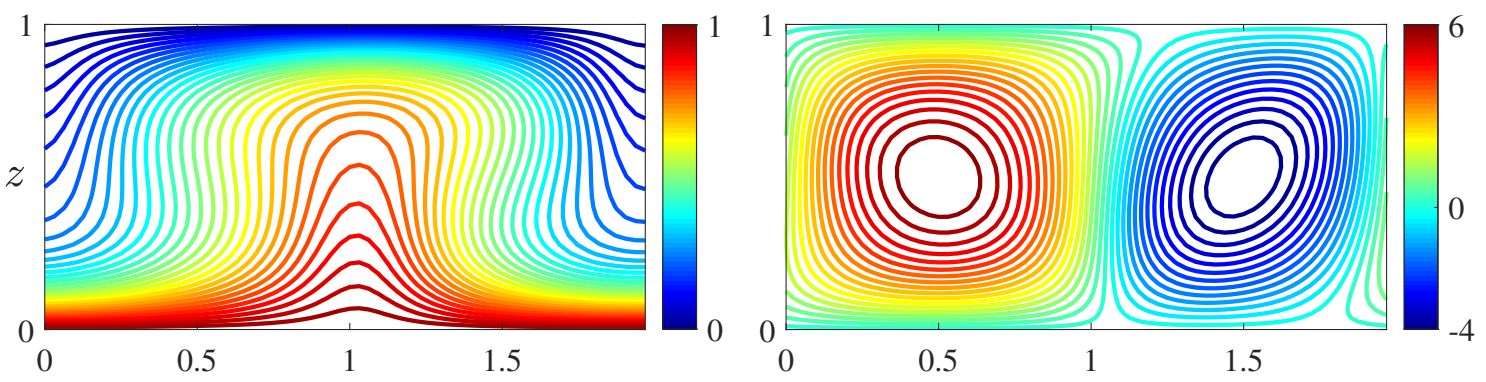

(b)
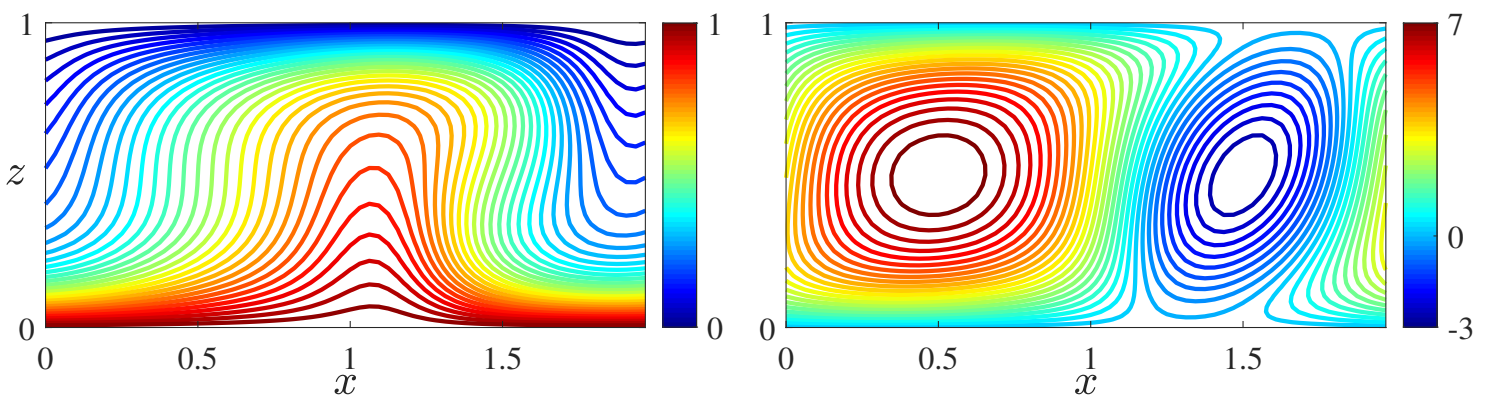

(c)

Figure 2. Snapshots of isotherms (left) and corresponding streamlines (right) from numerical simulations at $R a=100$ and $L=2$. (a) $\phi=0^{\circ}$; (b) $\phi=10^{\circ}$; (c) $\phi=25^{\circ}$. The streamlines of the natural (positive $\psi$ ) and antinatural (negative $\psi$ ) rolls are shown in red and blue, respectively. For a range of $\phi$ values, the flow takes the form of stable steady convective rolls. As $\phi$ is increased, the natural roll becomes more vigorous (see the colorbar limits) and more tightly attached to the walls, while the antinatural roll is suppressed and becomes detached from the walls. 

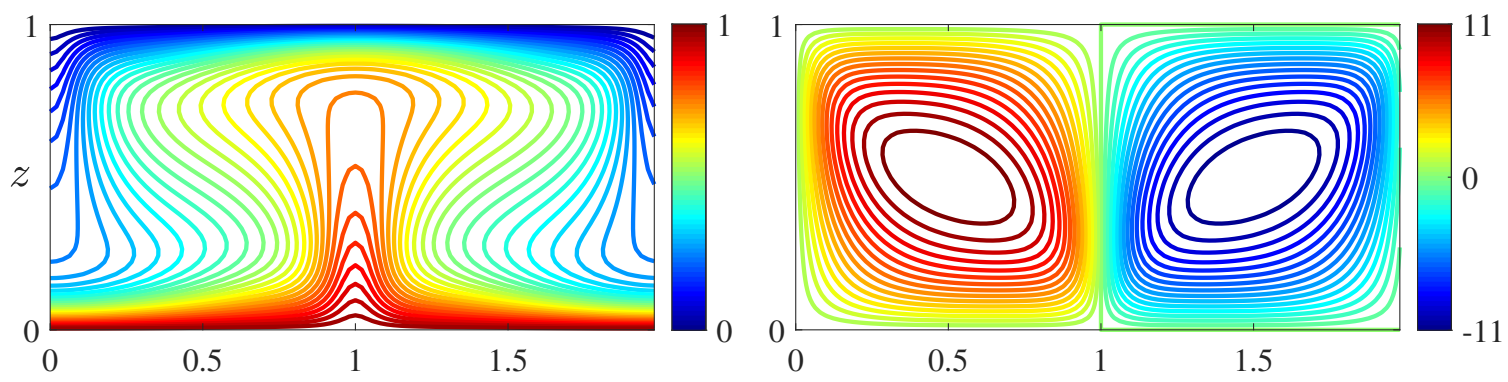

(a)
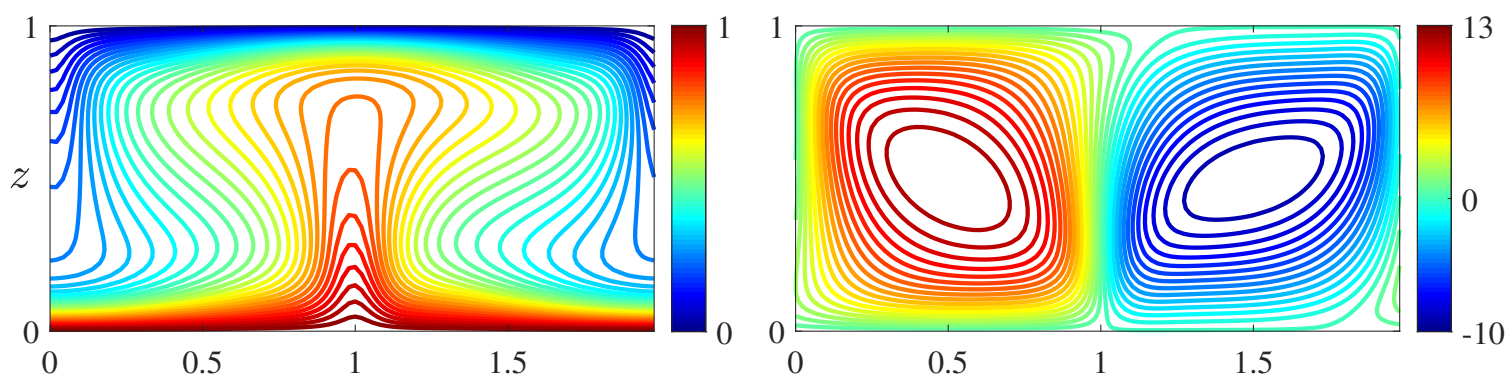

(b)
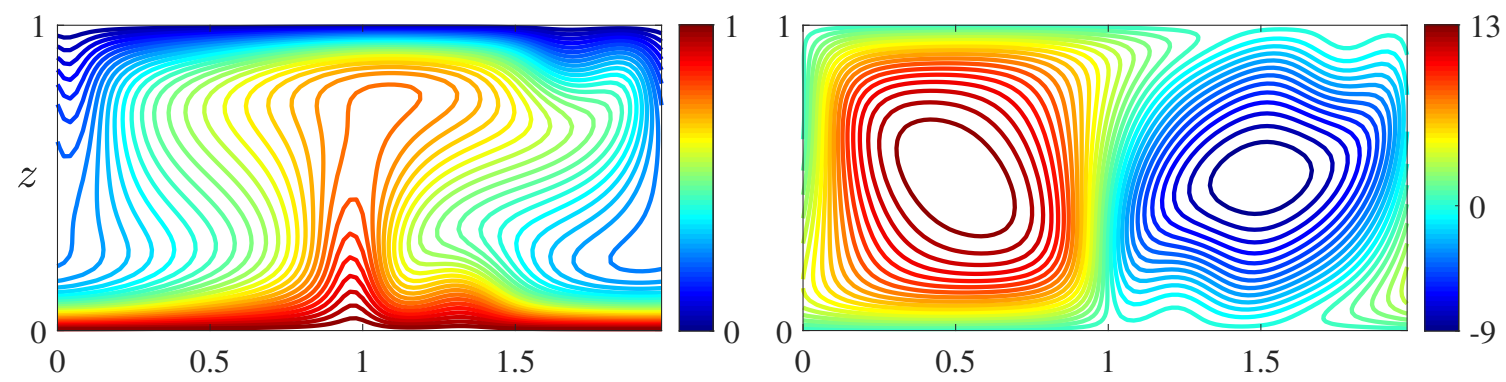

(c)
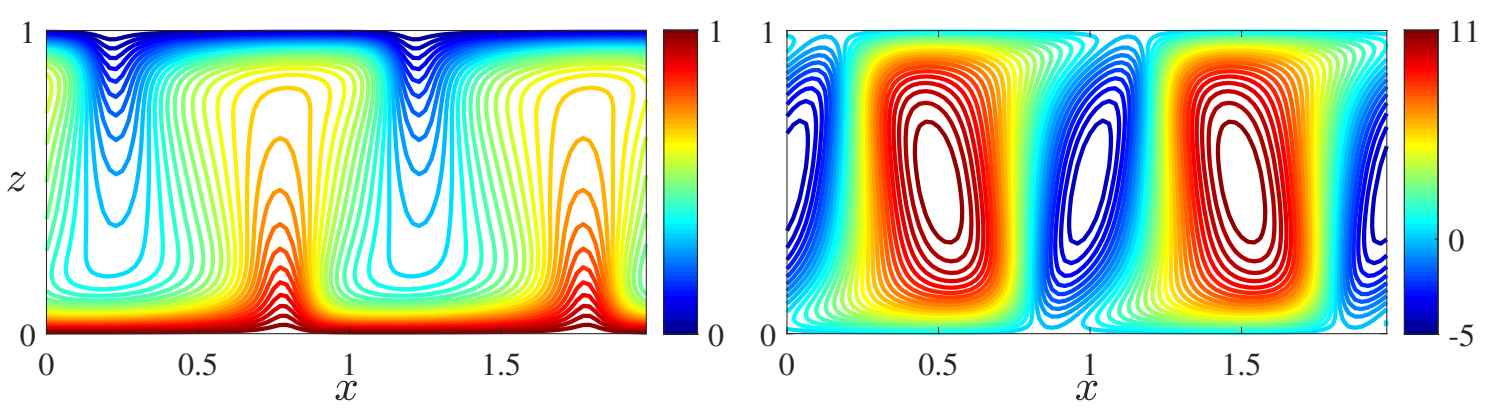

(d)

Figure 3. Snapshots of isotherms (left) and corresponding streamlines (right) from numerical simulations at $R a=300$ and $L=2$. (a) $\phi=0^{\circ}$; (b) $\phi=10^{\circ}$; (c) $\phi=17.5^{\circ}$; (d) $\phi=25^{\circ}$. In this case, the flows in $(\mathbf{a}, \mathbf{d})$ are steady; in $(\mathbf{b}, \mathbf{c})$, the upper and lower boundary layers of the antinatural rolls (negative $\psi$ ) become unstable. At $\phi \approx 25^{\circ}$, the small proto-plumes generated from the boundary-layer instabilities of the antinatural rolls split the unsteady two-cell convection (with one natural and one antinatural roll) into a steady four-cell convective state, thereby reducing the aspect ratio of each roll. 

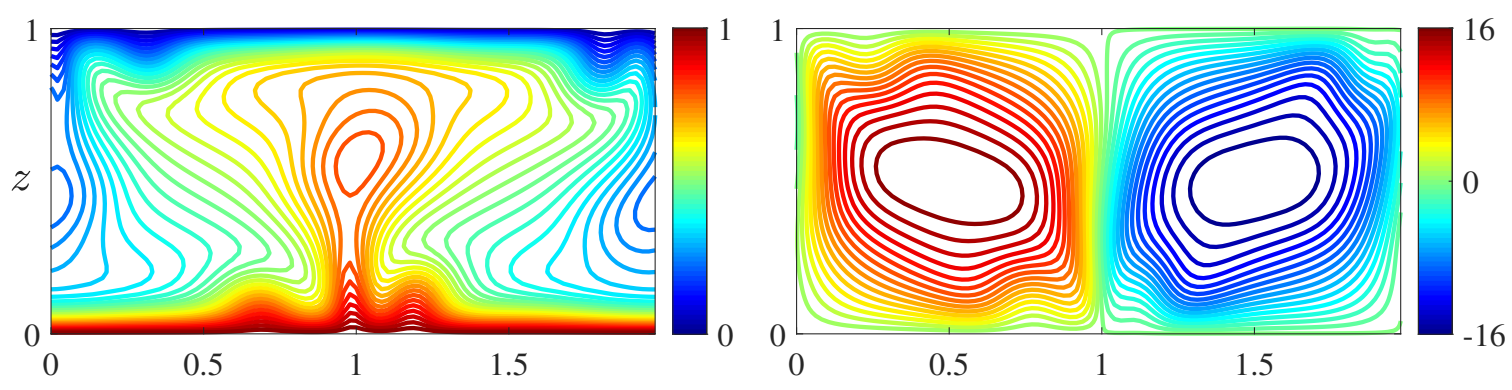

(a)
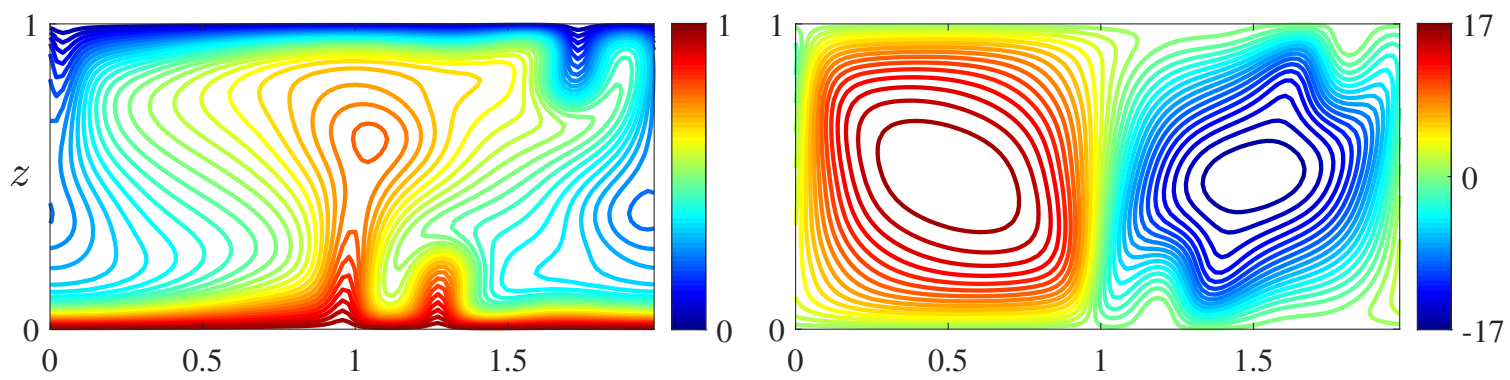

(b)
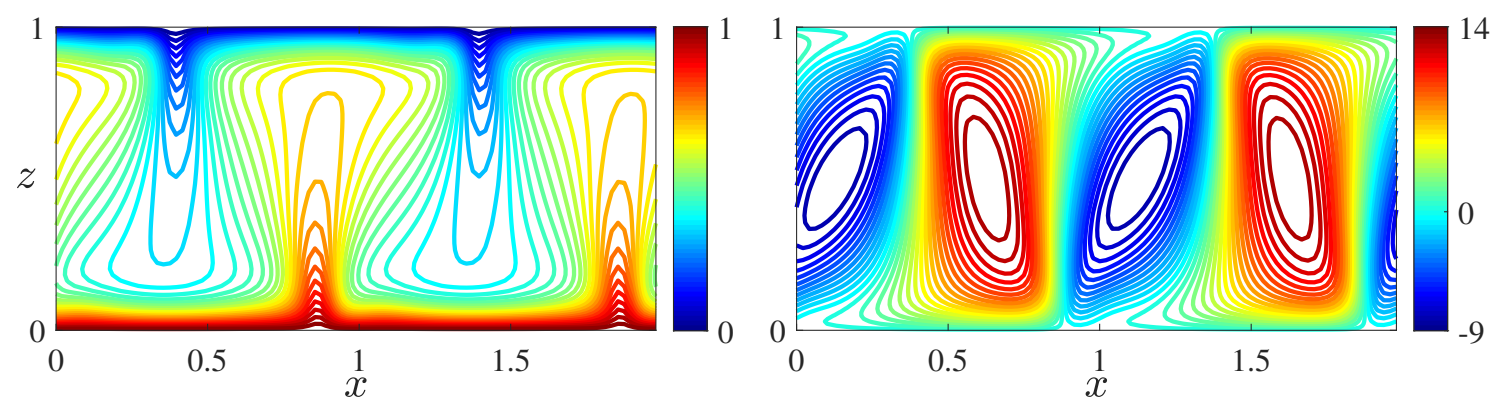

(c)

Figure 4. Snapshots of isotherms (left) and corresponding streamlines (right) from numerical simulations at $R a=500$ and $L=2$. (a) $\phi=0^{\circ}$; (b) $\phi=10^{\circ}$; (c) $\phi=15^{\circ}$. For $\phi<15^{\circ}$, the convection appears in the form of unsteady rolls $(\mathbf{a}, \mathbf{b})$. However, as $\phi$ is increased, the boundary-layer instability of the antinatural roll (negative $\psi$ ) becomes stronger and splits the unsteady two-cell convective state into the steady four-cell convection pattern shown in (c).

0
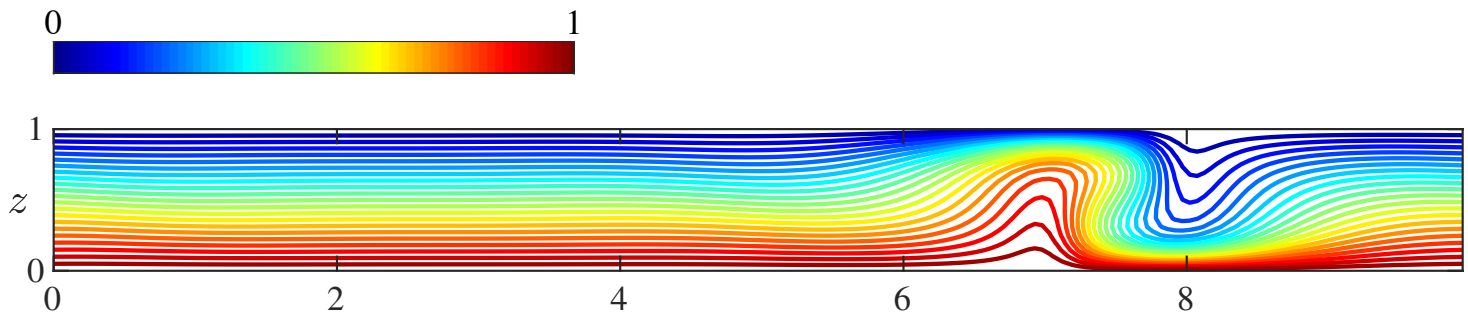

(a)

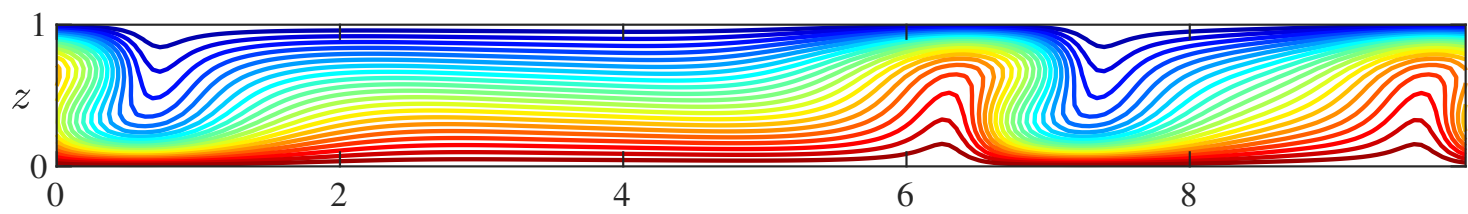

(b)

Figure 5. Cont. 


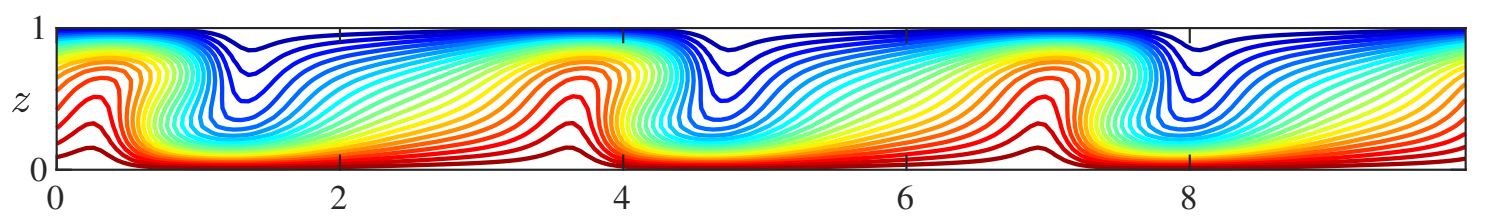

(c)

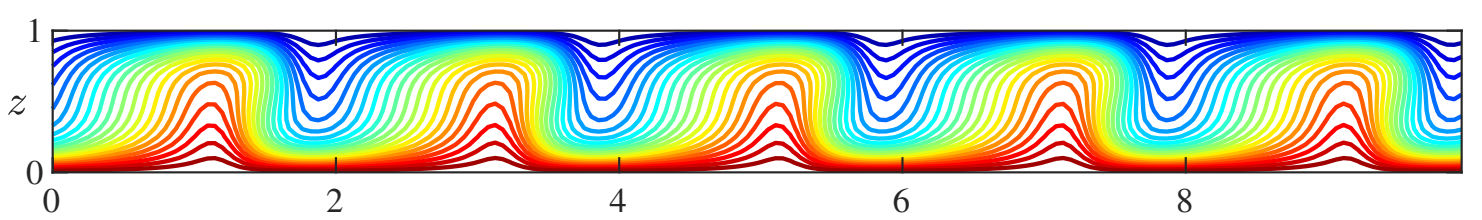

(d)

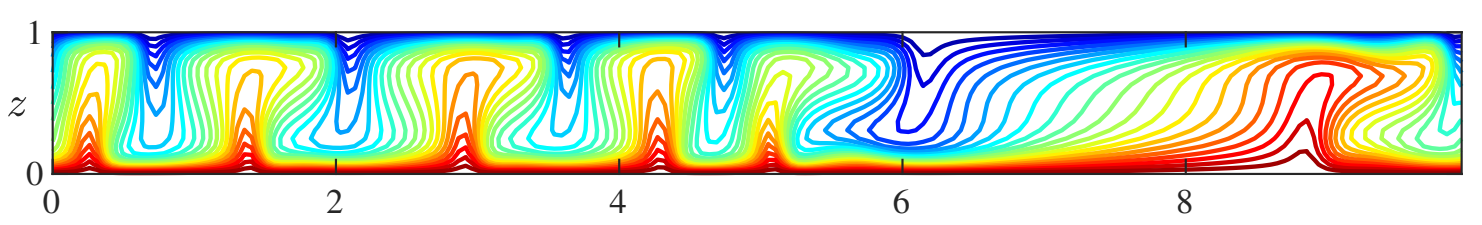

(e)

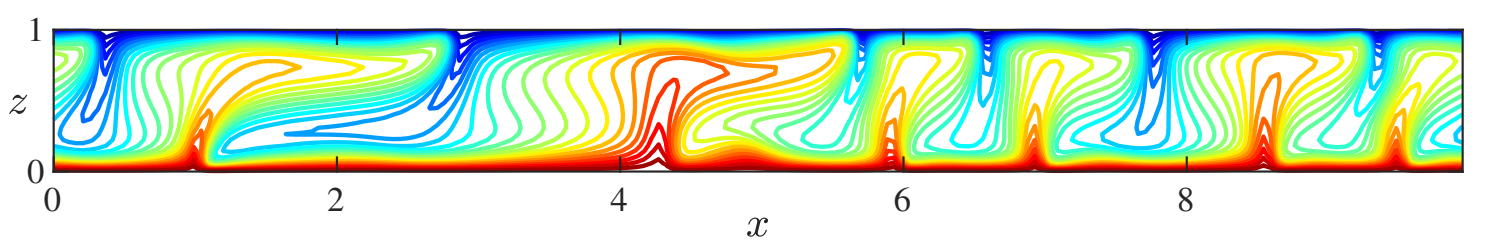

(f)

Figure 5. Snapshots of isotherms from numerical simulations at $\phi=35^{\circ}$ and $L=10$. (a-d) $R a=100$; (e) $R a=300 ;$ (f) $R a=500$. Sub-plots (a-d) show steady convective states obtained using different initial conditions, while (e) and (f) show snapshots of time-dependent states. Although the basic-state shear flow is linearly stable for $\phi>\phi_{t} \approx 31.3^{\circ}$ in $2 \mathrm{D}$, convection nevertheless may be realized by initializing with sufficiently large-amplitude disturbances; i.e., sub-critical instabilities are possible in this parameter regime. The spatially-localized convective states evident in $(\mathbf{a}, \mathbf{b})$, observed here for the first time in single-species porous medium convection, are one manifestation of this sub-critical instability.

In summary, our well-resolved numerical simulations show that the instantaneous flow at moderate $R a$ self-organizes into $O(1)$ aspect-ratio large-scale cellular flows, suggesting that the basic physics of inclined porous medium convection can be understood by studying the underlying exact coherent states, e.g., steady convective solutions, that support observed convective patterns. Accordingly, in the following sections, we compute steady convective solutions and then assess the stability of these nonlinear states.

\subsection{Steady Convective States}

We numerically compute steady solutions of Equations (5) and (6) using the Newton-GMRES (generalized minimal residuals) algorithm. Following Wen et al. [6] and Wen and Chini [36], we write the linear differential equations for the corrections as

$$
\left[\begin{array}{cc}
\nabla^{2} & R a\left(\cos \phi \partial_{x}-\sin \phi \partial_{z}\right) \\
-\partial_{x}+\theta_{z}^{i} \partial_{x}-\theta_{x}^{i} \partial_{z} & \nabla^{2}-\psi_{z}^{i} \partial_{x}+\psi_{x}^{i} \partial_{z}
\end{array}\right]\left[\begin{array}{c}
\triangle^{\psi} \\
\triangle^{\theta}
\end{array}\right]=\left[\begin{array}{c}
-F_{r e s}^{\psi} \\
-F_{r e s}^{\theta}
\end{array}\right]^{i}
$$

with the correction terms

$$
\triangle^{\psi}=\psi^{i+1}-\psi^{i}, \quad \Delta^{\theta}=\theta^{i+1}-\theta^{i},
$$


and the residuals of the steady governing equations

$$
\begin{aligned}
& F_{r e s}^{\psi}=\nabla^{2} \psi-R a\left(\partial_{z} \theta \sin \phi-\sin \phi-\partial_{x} \theta \cos \phi\right), \\
& F_{r e s}^{\theta}=\nabla^{2} \theta-\left(\partial_{z} \psi \partial_{x} \theta-\partial_{x} \psi \partial_{z} \theta+\partial_{x} \psi\right),
\end{aligned}
$$

where the superscript ' $i$ ' denotes the $i$ th Newton iterate. Then, (8) is solved using a Krylov-subspace (GMRES) iterative method under the centrosymmetry constraint for $\theta$. For each $i$, we stop the GMRES iteration once the L2-norm of the relative residual of (8) is less than $10^{-4}$, and finally stop the Newton iteration when the L2-norm of $\left(F_{r e s}^{\psi}, F_{r e s}^{\theta}\right)$ is less than $10^{-8}$. For each $R a$, the results from smaller $\phi$ are utilized as the initial conditions for simulations at larger $\phi$.

As noted above, steady convective states in an inclined porous layer are stable at small $R a$ (e.g., $R a \leq 200)$. However, as the Rayleigh number is increased, the boundary layers near the upper and lower walls become unstable and small-scale features are generated and advected around the cell by the large-scale roll (Figure 3c). In this section, the structure of these unstable steady convective states is investigated at $R a=500$ and $L=2$ for different inclination angles. As shown in Figure 6, the increasing inclination of the layer enhances the background flow, thereby intensifying the natural-roll motion and suppressing the antinatural-roll motion. Consequently, as $\phi$ is increased, the natural rolls become more vigorous and more tightly attached to the upper and lower walls; in contrast, the antinatural rolls become much weaker and detach from the walls (Figures 6 and 7).
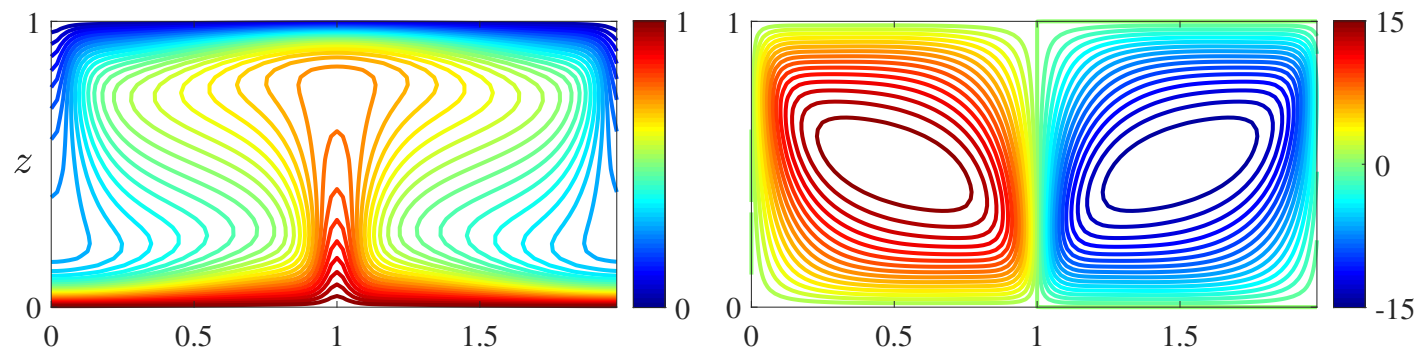

(a)
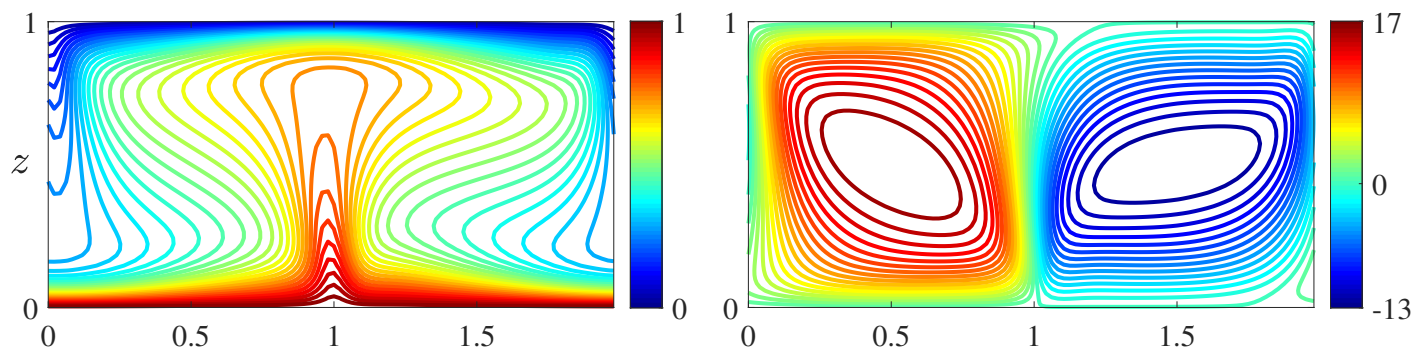

(b)
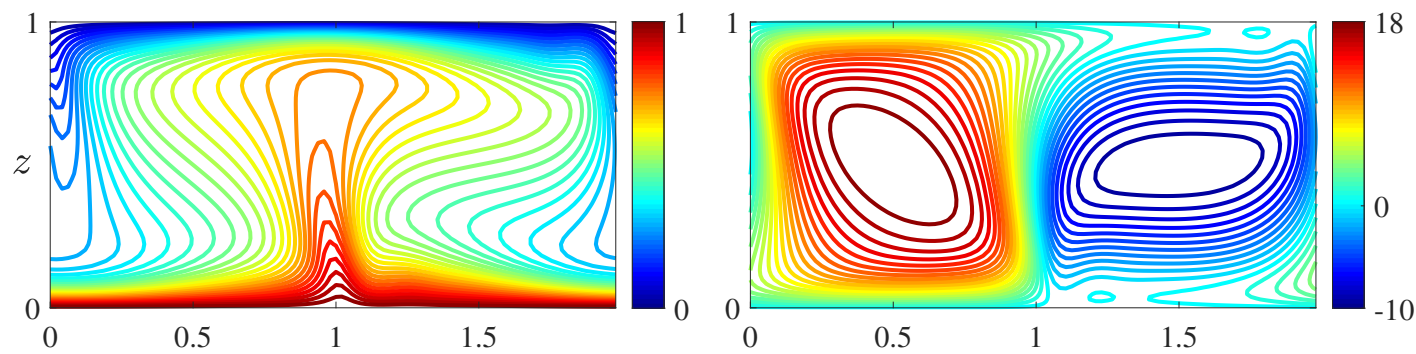

(c)

Figure 6. Cont. 

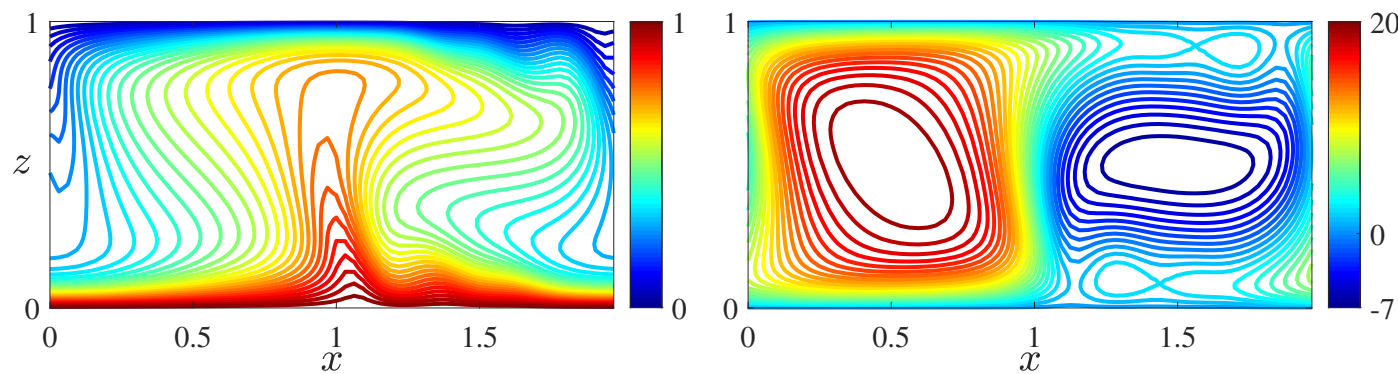

(d)

Figure 6. Isotherms (left) and streamlines (right) of steady convective states at $R a=500$ and $L=2$. (a) $\phi=0^{\circ}$; (b) $\phi=10^{\circ}$; (c) $\phi=20^{\circ}$; (d) $\phi=30^{\circ}$. As the inclination angle is increased, the natural roll (positive $\psi$ ) becomes more vigorous (see the colorbar limits) and more tightly attached to the walls, while the antinatural roll (negative $\psi$ ) is suppressed and becomes detached from the walls. At $\phi=30^{\circ}$, the antinatural roll makes contact with the upper and lower walls only at certain localized intervals in $x$.

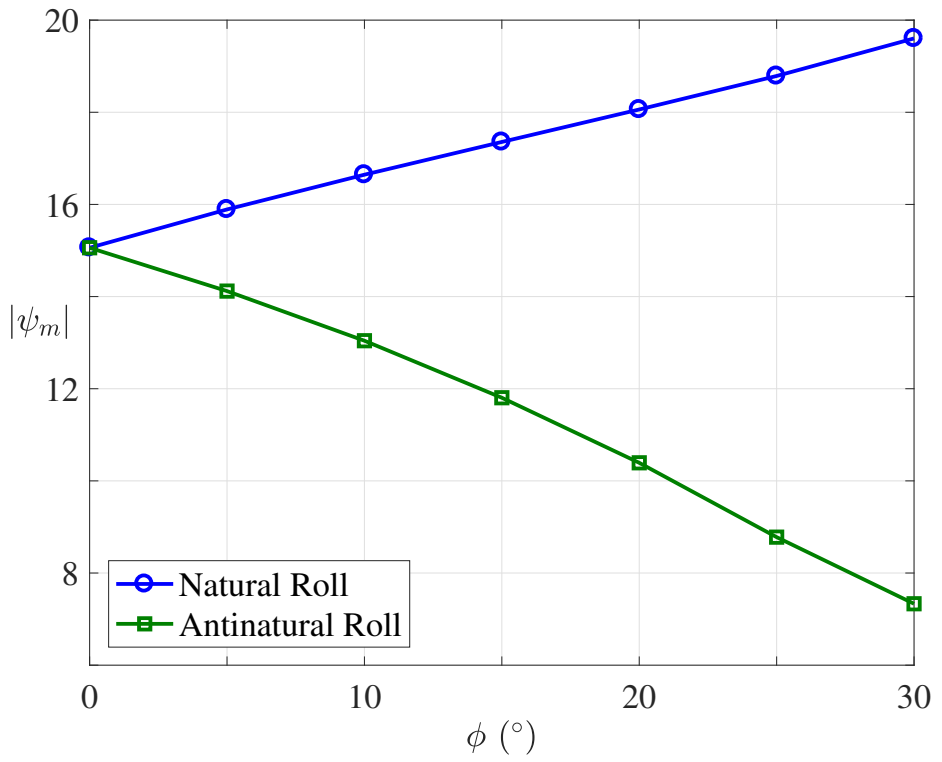

Figure 7. Magnitude of $\psi_{m}$ for steady convective states as a function of $\phi$ at $R a=500$ and $L=2$. $\psi_{m}$ denotes the extremum $\psi$ value corresponding to the natural roll with $\max (\psi)$ (positive) and antinatural roll with $\min (\psi)$ (negative). As $\phi$ is increased, the natural-roll motion is intensified, while the antinatural-roll motion is suppressed.

\subsection{Secondary Stability Analysis}

In this section, a spatial Floquet analysis is performed to investigate the linear stability of fully nonlinear steady convective states in an inclined porous layer. We decompose each field into a steady nonlinear 2D base flow (denoted with a subscript 's') plus a time-varying small-amplitude perturbation (denoted with a tilde),

$$
\begin{gathered}
\psi(\mathbf{x}, t)=\psi_{s}(\mathbf{x})+\tilde{\psi}(\mathbf{x}, t), \\
\theta(\mathbf{x}, t)=\theta_{s}(\mathbf{x})+\tilde{\theta}(\mathbf{x}, t) .
\end{gathered}
$$

Then, the evolution of the disturbances $\tilde{\psi}$ and $\tilde{\theta}$ are governed by following linearized equations

$$
\begin{gathered}
\nabla^{2} \tilde{\psi}=R a\left(\sin \phi \partial_{z}-\cos \phi \partial_{x}\right) \tilde{\theta} \\
\partial_{t} \tilde{\theta}=\nabla^{2} \tilde{\theta}-\partial_{x} \theta_{s} \partial_{z} \tilde{\psi}+\partial_{z} \theta_{s} \partial_{x} \tilde{\psi}+\partial_{x} \psi_{s} \partial_{z} \tilde{\theta}-\partial_{z} \psi_{s} \partial_{x} \tilde{\theta}-\partial_{x} \tilde{\psi} .
\end{gathered}
$$


According to Floquet theory, the solutions for the perturbations in (14) and (15) can be expressed as

$$
\left[\begin{array}{c}
\tilde{\theta} \\
\tilde{\psi}
\end{array}\right]=e^{i \beta k_{s} x}\left\{\sum_{n=-\infty}^{\infty}\left[\begin{array}{c}
\hat{\hat{\theta}}_{n}(z) \\
\hat{\tilde{\psi}}_{n}(z)
\end{array}\right] e^{i n k_{s} x}\right\} e^{\lambda t}+\text { c.c., }
$$

where $\lambda$ is the temporal growth rate, $k_{s}$ is the fundamental wavenumber of the spatially-periodic steady solution, $n$ is the wall-parallel Fourier mode number, $\beta$ is the real Floquet parameter $(0 \leq \beta \leq 0.5)$, which provides the freedom to modify the fundamental horizontal wavenumber of the perturbation, and c.c. denotes complex conjugate. Substituting the ansatz (16) into Equations (14) and (15) yields

$$
\begin{array}{r}
-R a\left[\sin \phi \partial_{z}-i(n+\beta) k_{s} \cos \phi\right] \hat{\hat{\theta}}_{n}+\left[\partial_{z}^{2}-(n+\beta)^{2} k_{s}^{2}\right] \hat{\tilde{\psi}}_{n}=0, \\
{\left[\partial_{z}^{2}-(n+\beta)^{2} k_{s}^{2}+\tilde{h}_{n}\right] \hat{\tilde{\theta}}_{n}+\left[-i(n+\beta) k_{s}+\tilde{g}_{n}\right] \hat{\tilde{\psi}}_{n}=\lambda \hat{\tilde{\theta}}_{n}}
\end{array}
$$

for each $n$, where $\tilde{h}_{n}$ and $\tilde{g}_{n}$ can be determined numerically by calculating the convolution of the non-constant-coefficient terms $\left(\partial_{x} \psi_{s} \partial_{z}-\partial_{z} \psi_{s} \partial_{x}\right)$ and $\left(-\partial_{x} \theta_{s} \partial_{z}+\partial_{z} \theta_{s} \partial_{x}\right)$, respectively, in (15). Finally, the eigenvalue problem (17) and (18) is discretized using a Chebyshev collocation method and the resulting algebraic eigenvalue problem is solved using Arnoldi iteration to obtain the leading eigenvalues and eigenfunctions.

Our results reveal that, at moderate $R a$, the maximum convective growth rate $\sigma_{m} \equiv \operatorname{Re}\left\{\lambda_{m}\right\} / R a$ for both the horizontal and inclined cases is independent of $\beta$, and the corresponding fastest-growing eigenfunction shares a similar spatial structure for different $\beta$. Hence, below we only present the results of our stability analysis at $\beta=0$. Figure 8 shows the variation of $\sigma_{m}$ as a function of $\phi$ at the aspect ratio $L_{s}=2 \pi / k_{s}=2$. The inclination of the layer enhances the instability of the steady state, and for each $R a$, there exists a peak in $\sigma_{m}$ at particular angle $\phi_{m}$. [Note that in our time-dependent numerical simulations the increasing instability with $\phi$ generally causes the two-cell convection pattern to split into a four-cell pattern before $\phi_{m}$ is reached (Table 1 and Figure 8).] Interestingly, this trend is opposite to that exhibited by the basic state itself; that is, increasing the inclination of the layer stabilizes the basic state, primarily by reducing the destabilizing effect of buoyancy, while destabilizing steady convective states (at least for $\phi \leq \phi_{m}$ ) owing largely to the intensification of the background shear flow. Moreover, the structure of the most unstable eigenfunction shown in Figure 9 and the results presented in Figure 10 confirm that the antinatural rolls are more unstable than are the natural rolls at moderate Rayleigh number, as also indicated by the numerical simulations in Section 3.1. Again, as $\phi$ is increased, the natural roll of the steady state strengthens and becomes more tightly attached to the walls, and thereby is stabilized; on the contrary, the antinatural roll is suppressed and becomes detached from the walls, and thereby is destabilized. Thus, the increase in the maximum growth rate $\sigma_{m}$ with $\phi$ in Figure 8 is attributable to the destabilization of the antinatural roll. 


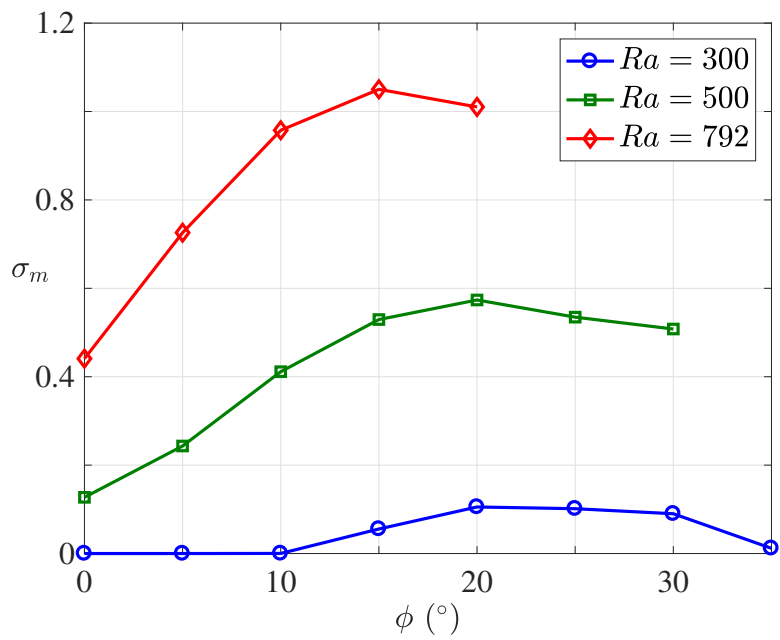

Figure 8. Variation of the maximum growth rate, $\sigma_{m}$, with $\phi$ at moderate $R a, L_{s}=2$ and $\beta=0$. At $R a=300$, the steady state is marginally stable for $\phi<10^{\circ}$ and becomes weakly unstable at $\phi=10^{\circ}$. The same branch of steady states is not obtained at large $\phi$ for $R a=500$ and 792 using the present numerical scheme.

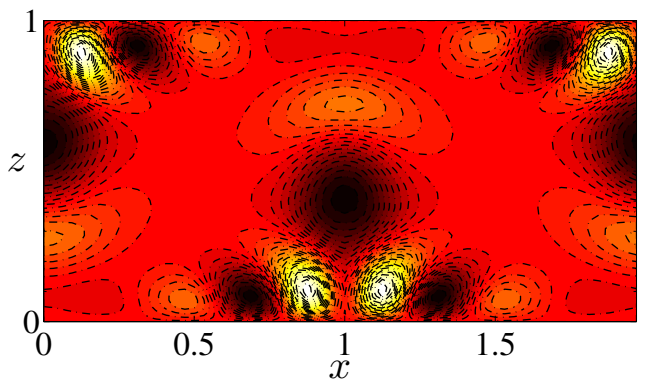

(a)

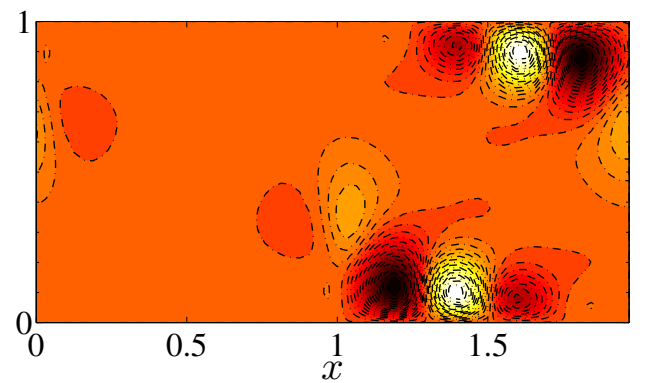

(b)

Figure 9. The fastest-growing $2 \mathrm{D}$ temperature eigenfunctions at $R a=500, L_{s}=2$ and $\beta=0$. (a) $\phi=0^{\circ}$; (b) $\phi=20^{\circ}$. For the horizontal case, reflection symmetry is satisfied and both the natural and antinatural rolls are equally unstable. However, as $\phi$ is increased, the natural roll is stabilized and the instability of the antinatural roll is intensified.

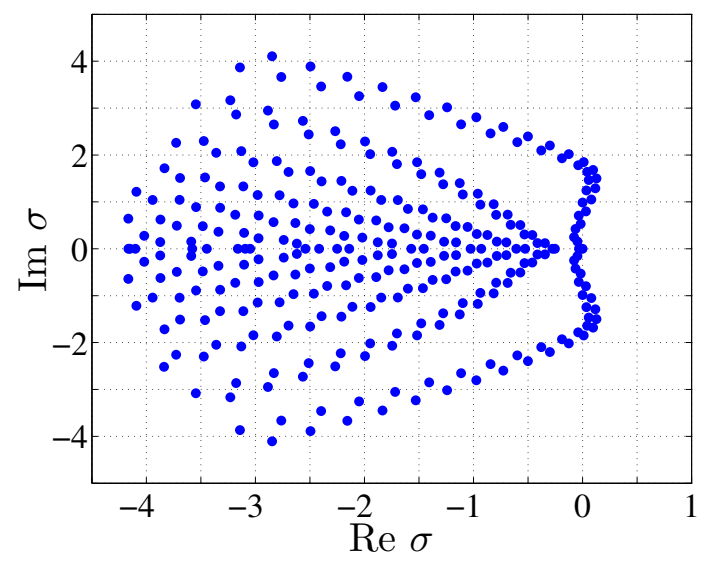

(a)

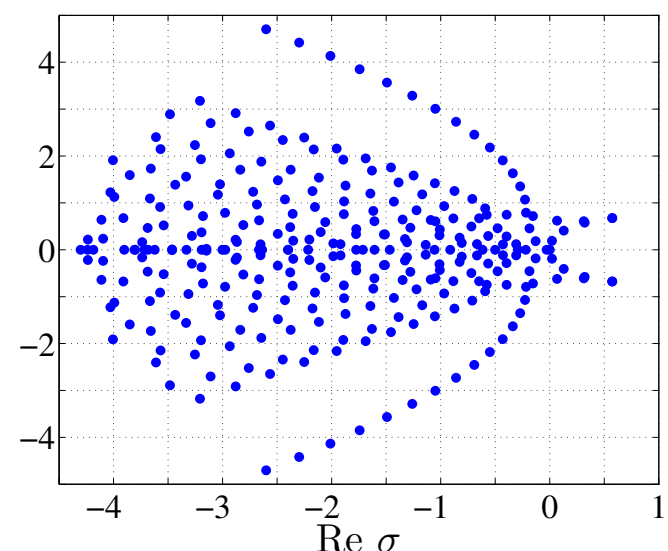

(b)

Figure 10. The leading eigenvalues, $\sigma=\lambda / R a$, at $R a=500, L_{s}=2$ and $\beta=0$. (a) $\phi=0^{\circ} ;(\mathbf{b}) \phi=20^{\circ}$. All of the unstable modes for both the horizontal and inclined cases exhibit a similar structure as that of the corresponding fastest-growing mode in Figure 9. 


\section{Conclusions}

In this study, we investigate the flow structure and dynamics of moderate- $R a$ convection in an inclined 2D porous layer uniformly heated from below. Using pseudospectral numerical simulations, we show the evolution of the $O(1)$ aspect-ratio large-scale cellular flows as functions of $R a$ and $\phi$. Our numerical simulation results indicate that the inclination of the layer breaks the reflection symmetry of the convective rolls in the wall-parallel direction. As the inclination angle $\phi$ is increased, the background shear flow strengthens, thereby intensifying the natural-roll motions and suppressing the antinatural-roll motions. Therefore, for increasing Rayleigh number $R a$ and at sufficiently large $\phi$, the boundary layers of the antinatural roll become unstable prior to those of the natural roll. Interestingly, our numerical simulations reveal for the first time the existence of spatially-localized convective states in single-species porous medium convection at large $\phi$, which may be anticipated based on the gap in parameter values for linear and nonlinear stability of the basic state [36].

To better understand the physics of inclined porous medium convection at different $\phi$, we also analyze the structure and stability of steady nonlinear convective states at moderate $R a$. We compute the steady solutions using a Newton-GMRES algorithm and then perform secondary stability analysis using Floquet theory. Consistent with the unsteady flow observed in our numerical simulations, the steady states appear in the form of large-scale convective rolls: one natural roll rotating in a counterclockwise direction; and one antinatural roll rotating in a clockwise direction. As the inclination angle is increased, the strengthening background mean flow enhances the motion of the natural roll causing it to more tightly attach to the upper and lower walls, but weakens the motion of the antinatural roll driving detachment from the walls, at least for sufficiently large $\phi$. Moreover, Floquet analysis of these steady states reveals that before the antinatural roll is completely detached from the walls, the inclination of the layer stabilizes the boundary layers of the natural roll, but intensifies the boundary-layer instability of the antinatural roll. These analyses shed light on the development of moderate- $R a$ large-scale cellular flows at different inclination angles.

Author Contributions: Conceptualization, B.W. and G.P.C.; methodology, B.W. and G.P.C.; software, B.W.; validation, B.W.; formal analysis, B.W. and G.P.C.; investigation, B.W. and G.P.C.; resources, G.P.C.; data curation, B.W.; writing - original draft preparation, B.W. and G.P.C.; writing-review and editing, B.W. and G.P.C.; visualization, B.W.; supervision, G.P.C.; project administration, G.P.C.; funding acquisition, G.P.C.

Funding: This work was funded by NSF Award DMS-0928098 (G.P.C.).

Acknowledgments: We thank Edgar Knobloch for helpful discussions and the referees for their careful review of the manuscript.

Conflicts of Interest: The authors declare no conflict of interest.

\section{References}

1. Horton, C.W.; Rogers, F.T. Convection currents in a porous medium. J. Appl. Phys. 1945, 16, 367-370. [CrossRef]

2. Lapwood, E.R. Convection of a fluid in a porous medium. Proc. Camb. Phil. Soc. 1948, 44, 508-521. [CrossRef]

3. Graham, M.D.; Steen, P.H. Strongly interacting traveling waves and quasiperiodic dynamics in porous medium convection. Phys. D 1992, 54, 331-350. [CrossRef]

4. Otero, J.; Dontcheva, L.A.; Johnston, H.; Worthing, R.A.; Kurganov, A.; Petrova, G.; Doering, C.R. High-Rayleigh-number convection in a fluid-saturated porous layer. J. Fluid Mech. 2004, 500, 263-281. [CrossRef]

5. Hewitt, D.R.; Neufeld, J.A.; Lister, J.R. Ultimate regime of high Rayleigh number convection in a porous medium. Phys. Rev. Lett. 2012, 108, 224503. [CrossRef] [PubMed]

6. Wen, B.; Corson, L.T.; Chini, G.P. Structure and stability of steady porous medium convection at large Rayleigh number. J. Fluid Mech. 2015, 772, 197-224. [CrossRef]

7. Liang, Y.; Wen, B.; Hesse, M.; DiCarlo, D. Effect of dispersion on solutal convection in porous media. Geophys. Res. Lett. 2018, 45, 9690-9698. [CrossRef] 
8. Metz, B.; Davidson, O.; de Coninck, H.; Loos, M.; Meyer, L. IPCC Special Report on Carbon Dioxide Capture and Storage; Cambridge University Press: New York, NY, USA, 2005.

9. Riaz, A.; Hesse, M.; Tchelepi, H.A.; Orr, F.M. Onset of convection in a gravitationally unstable diffusive boundary layer in porous media. J. Fluid Mech. 2006, 548, 87-111. [CrossRef]

10. Nield, D.A.; Bejan, A. Convection in Porous Media, 4th ed.; Springer: New York, NY, USA, 2013.

11. Phillips, O.M. Geological Fluid Dynamics: Sub-Surface Flow and Reactions; Cambridge University Press: Cambridge, UK, 2009.

12. Schubert, G.; Straus, J.M. Transitions in time-dependent thermal convection in fluid-saturated porous media. J. Fluid Mech. 1982, 121, 301-313. [CrossRef]

13. Kimura, S.; Schubert, G.; Straus, J.M. Route to chaos in porous-medium thermal convection. J. Fluid Mech. 1986, 166, 305-324. [CrossRef]

14. Kimura, S.; Schubert, G.; Straus, J.M. Instabilities of steady, periodic, and quasi-periodic modes of convection in porous media. J. Heat Transf. 1987, 109, 350-355. [CrossRef]

15. Aidun, C.K.; Steen, P.H. Transition to oscillatory convective heat transfer in a fluid-saturated porous medium. J. Thermophys. Heat Transf. 1987, 1, 268-273.

16. Graham, M.D.; Steen, P.H. Plume formation and resonant bifurcations in porous-media convection. J. Fluid Mech. 1994, 272, 67-90. [CrossRef]

17. Wen, B.; Chini, G.P.; Dianati, N.; Doering, C.R. Computational approaches to aspect-ratio-dependent upper bounds and heat flux in porous medium convection. Phys. Lett. A 2013, 377, 2931-2938. [CrossRef]

18. MacMinn, C.W.; Juanes, R. Buoyant currents arrested by convective dissolution. Geophys. Res. Lett. 2013, 40, 2017-2022. [CrossRef]

19. Tsai, P.A.; Riesing, K.; Stone, H.A. Density-driven convection enhanced by an inclined boundary: Implications for geological $\mathrm{CO}_{2}$ storage. Phys. Rev. E 2013, 87, 011003. [CrossRef]

20. Sathaye, K.; Hesse, M.; Cassidy, M.; Stockli, D. Constraints on the magnitude and rate of $\mathrm{CO}_{2}$ dissolution at Bravo Dome natural gas field. Proc. Nat. Acad. Sci. USA 2014, 111, 15332-15337. [CrossRef]

21. Akhbari, D.; Hesse, M.A. Causes of underpressure in natural $\mathrm{CO}_{2}$ reservoirs and implications for geological storage. Geology 2017, 45, 47-50. [CrossRef]

22. Bories, S.A.; Combarnous, M.A.; Jaffrenou, J. Observations des différentes formes d'écoulements thermoconvectifs dans une couche poreuse inclinée. CR Acad. Sci. Paris A 1972, 275, 857-860.

23. Bories, S.A.; Monferran, L. Condition de stabilité et échange thermique par convection naturelle dans une couche poreuse inclinée de grande extension. CR Acad. Sci. Paris B 1972, 274, 4-7.

24. Bories, S.A.; Combarnous, M.A. Natural convection in a sloping porous layer. J. Fluid Mech. 1973, 57, 63-79. [CrossRef]

25. Caltagirone, J.P.; Cloupeau, M.; Combarnous, M. Convection naturelle fluctuante dans une couche poreuse horizontale. CR Acad. Sci. Paris B 1971, 273, 833-836.

26. Kaneko, T. An Experimental Investigation of Natural Convection in Porous Media. Master's Thesis. University of Calgary, Calgary, AB, Canada, 1972.

27. Kaneko, T.; Mohtadi, M.F.; Aziz, K. An experimental study of natural convection in inclined porous media. Int. J. Heat Mass Transf. 1974, 17, 485-496. [CrossRef]

28. Caltagirone, J.P.; Bories, S. Solutions and stability criteria of natural convective flow in an inclined porous layer. J. Fluid Mech. 1985, 155, 267-287. [CrossRef]

29. Rees, D.A.S.; Bassom, A.P. The onset of Darcy-Bénard convection in an inclined layer heated from below. Acta Mech. 2000, 144, 103-118. [CrossRef]

30. Rees, D.A.S.; Postelnicu, A. The onset of convection in an inclined anisotropic porous layer. Int. J. Heat Mass Transf. 2001, 44, 4127-4138. [CrossRef]

31. Rees, D.A.S.; Storesletten, L.; Postelnicu, A. The onset of convection in an inclined anisotropic porous layer with oblique principle axes. Transp. Porous Med. 2006, 62, 139-156. [CrossRef]

32. Rees, D.A.S.; Barletta, A. Linear instability of the isoflux Darcy-Bénard problem in an inclined porous layer. Transp. Porous Med. 2011, 87, 665-678. [CrossRef]

33. Barletta, A.; Storesletten, L. Thermoconvective instabilities in an inclined porous channel heated from below. Int. J. Heat Mass Transf. 2011, 54, 2724-2733. [CrossRef]

34. Barletta, A.; Rees, D.A.S. Local thermal non-equilibrium analysis of the thermoconvective instability in an inclined porous layer. Int. J. Heat Mass Transf. 2015, 83, 327-336. [CrossRef] 
35. Celli, M.; Barletta, A. Onset of buoyancy driven convection in an inclined porous layer with an isobaric boundary. Int. J. Heat Mass Transf. 2019, 132, 782-788. [CrossRef]

36. Wen, B.; Chini, G.P. Inclined porous medium convection at large Rayleigh number. J. Fluid Mech. 2018, 837, 670-702. [CrossRef]

37. Walch, J.; Dulieu, B. Convection de Rayleigh-Béenard dans une cavité poreuse faiblement inclinée. J. Phys. Lett. 1982, 43, 103-107. [CrossRef]

38. Moya, S.L.; Ramos, E.; Sen, M. Numerical study of natural convection in a tilted rectangular porous material. Int. J. Heat Mass Transfer 1987, 30, 741-756. [CrossRef]

39. Sen, M.; Vasseur, P.; Robillard, L. Multiple steady states for unicellular natural convection in an inclined porous layer. Int. J. Heat Mass Transf. 1987, 30, 2097-2113. [CrossRef]

40. Shi, Z.; Wen, B.; Hesse, M.; Tsotsis, T.; Jessen, K. Measurement and modeling of $\mathrm{CO}_{2}$ mass transfer in brine at reservoir conditions. Adv. Water Resour. 2018, 113, 100-111. [CrossRef]

41. Wen, B.; Akhbari, D.; Zhang, L.; Hesse, M.A. Convective carbon dioxide dissolution in a closed porous medium at low pressure. J. Fluid Mech. 2018, 854, 56-87. [CrossRef]

42. Wen, B.; Chang, K.W.; Hesse, M.A. Rayleigh-Darcy convection with hydrodynamic dispersion. Phys. Rev. Fluids 2018, 3, 123801. [CrossRef]

43. Boyd, J.P. Chebyshev and Fourier Spectral Methods, 2nd ed.; Dover: New York, NY, USA, 2000.

44. Nikitin, N. Third-order-accurate semi-implicit Runge-Kutta scheme for incompressible Navier-Stokes equations. Int. J. Numer. Meth. Fluids 2006, 51, 221-233. [CrossRef]

45. Peyret, R. Spectral Methods for Incompressible Viscous Flow; Springer: New York, NY, USA, 2002.

46. Lo Jacono, D.; Bergeon, A.; Knobloch, E. Spatially localized binary fluid convection in a porous medium. Phys. Fluids 2010, 22, 909. [CrossRef]

47. Lo Jacono, D.; Bergeon, A.; Knobloch, E. Three-dimensional spatially localized binary-fluid convection in a porous medium. J. Fluid Mech. 2013, 730, R2. [CrossRef]

(C) 2019 by the authors. Licensee MDPI, Basel, Switzerland. This article is an open access article distributed under the terms and conditions of the Creative Commons Attribution (CC BY) license (http:/ / creativecommons.org/licenses/by/4.0/). 